\title{
Predicting Articular Cartilage Behavior with a Non-Linear Microstructural Model
}

\author{
Fulin Lei and Andras Z. Szeri ${ }^{*}$ \\ Center for Biomedical Engineering Research, Department of Mechanical Engineering, University of Delaware, Newark, \\ $U S A$
}

\begin{abstract}
We report here on a non-linear poroelastic model for the mechanical response of collagenous soft tissues such as articular cartilage. The tissue consists of a porous, fibril-reinforced, hyperelastic solid, saturated with an incompressible fluid, and Darcy's law governs solid-fluid interaction. The solid matrix is characterized by the isotropic hyperfoam strain energy function and its permeability is made to depend on local strain. The fibrils are non-linear, provide tensile stiffness only, exhibit viscoelasticity and have arbitrary, three-dimensional, statistical distributions. The stress tensor in the fibril network is calculated from the constitutive law for a single fibril with the aid of the fibril distribution functions. With a specific viscoelastic fibril constitutive relationship and a three-layered cartilage construction, the model is shown to predict well strain-dependent and time-dependent behavior in unconfined compression and tension and in unconfined compression and indentation, using identical sets of material parameters.
\end{abstract}

Keywords: Cartilage, finite deformation, poroelasticity, fibril reinforcement, hyperfoam.

\section{INTRODUCTION}

Articular cartilage is a smooth, thin tissue that covers the ends of articulating bones of diarthrodial joints. The unique mechanical properties of healthy cartilage enable the joints to bear large, varying loads while maintaining almost friction-free movement [1]. The mechanical modeling of articular cartilage is a prerequisite for our understanding the biophysical functions of synovial joints $[2,3]$.

Due to the complexity of mechanical response of articular cartilage, numerous constitutive models have been developed over the past several decades [4-10], with varying degrees of success. Two different approaches, namely macroscopic and microstructural, have been used to describe the material behavior of the tissue. Macroscopic models view the solid phase as a single material whose mechanical properties are based on bulk behavior [5, 7, 9, 11-13]. In contrast, microstructural models consider the solid phase to be a composite whose properties depend on the structure and the properties of its components [10, 14-17]. There also exist micromechanical models for fiber composites [18-21], and microstructural models for the drained elastic properties of soft tissues [22-25].

Two of the salient features of the cartilage's mechanical response are poroelasticity, i.e., flow-dependent viscoelastic behavior, and tension-compression nonlinearity. All biphasic and consolidation models account for the first of these, and the phenomena of small compressive modulus and large tensile modulus are predictable by the bimodular biphasic model [12] and the fibril-reinforced biphasic model [10]. There are, however, other, less prominent, characteristics of cartilage response that should also be accounted for. Some researchers hold, for example, that inherent viscoelasticity of the drained tissue plays an important role [6, 15, 16, 26, 27].

*Address correspondence to this author at the Center for Biomedical Engineering Research, Department of Mechanical Engineering, University of Delaware, Newark, USA; E-mail: szeri@ me.udel.edu
Strain-hardening has been observed in both compression [28, 29] and tension [30, 31], while the tensile Poisson's ratio has been shown to remain approximately constant [31, 32]. The combination of bimodular model and quasi-linear viscoelasticity will describe stress-relaxation in both compression and tension [27]; the stress relaxation may also be explained through incorporating fibril viscoelasticity into the fibrilreinforced biphasic model [16]. Wilson and coworkers further developed fibril-reinforced poroelastic models by including a more realistic network of fibrils $[15,33]$.

In a previous paper [17], we have reported on a general microstructural poroelastic model that could incorporate arbitrary fibril distribution and thus explain the anomaly that small modulus and Poisson's ratio are observed in unconfined compression, while large modulus and lateral Poisson's ratio govern tension. This model has its limitations, however: fibril viscoelasticity and nonlinearity are not included, and fibril distribution does not change with deformation. In consequence, strain-dependent properties of cartilage cannot be explained by that model.

The present study removes some of the limitations of our earlier model, thus permitting clarification of various additional cartilage properties. We hypothesize that the inclusion of hyperelasticity for the solid matrix, and nonlinearity and viscoelasticity for the fibrils into our microstructural poroelastic model [17] will result in a comprehensive approach that may be able to explain the most essential mechanical properties of articular cartilage. To demonstrate the predictive capability of this new model, we employ it to analyze strain-dependent equilibrium properties and curve-fit experimental stress-relaxation data of cartilage samples in unconfined compression, tension and indentation tests.

\section{METHODS}

\subsection{Model Formulations}

We envisage the cartilage tissue as a saturated mixture of a porous solid matrix (identifier $s$ ), a fluid (identifier $w$ ), and 
a fibril network (identifier $f$ ) embedded in the solid matrix. The volume fractions of solid matrix, fibril network and interstitial fluid are denoted by $\phi^{s}, \phi^{f}$ and $\phi^{w}$ respectively. The governing equations are identical to those of biphasic theories [5, 34], except for the constitutive equation for the solid phase. In our composite view of the cartilage, the threedimensional fibril network reinforces the hyperelastic solid matrix and the Cauchy stress tensor of the tissue is given by

$$
\mathbf{T}=-p \mathbf{I}+\frac{2}{J} \mathbf{F} \frac{\partial W}{\partial \mathbf{C}} \mathbf{F}^{T}+\mathbf{T}^{f}
$$

Here $\mathbf{F}$ is the deformation gradient tensor and $J$ is its Jacobian determinant, $W$ is the strain energy function, $\mathbf{C}=\mathbf{F}^{T} \mathbf{F}$ is the right Cauchy-Green tensor, $p$ is the interstitial fluid pressure and $\mathbf{T}^{f}$ is the stress tensor in the fibril network.

To calculate the elastic stress arising from the deformation of the fibril network, we make the following assumptions: the fibrils are locally straight and support tensile load only; bonding between solid matrix and fibril is perfect and constrains perfectly against slippage; interaction between fibrils is neglected.

We characterize fibril configuration, at any stage of the (large) deformation by the fibril distribution function $f(\phi, \theta)$, which has reference value $f_{0}(\Phi, \Theta)$. Here $(\phi, \theta)$ and $(\Phi, \Theta)$ are Eulerian angles and their initial values, respectively [17, 35]. The relationship between initial and current configurations is determined by the deformation gradient $\mathbf{F}$, which also rotates the initial fibril orientation

$\mathbf{n}_{0}=(\sin \Theta \cos \Phi, \sin \Theta \sin \Phi, \cos \Theta)$

into the new fibril direction $\mathbf{n}$

$\mathbf{n}=\frac{\mathbf{F} \mathbf{n}_{0}}{\left|\mathbf{F} \mathbf{n}_{0}\right|}=(\sin \theta \cos \phi, \sin \theta \sin \phi, \cos \theta)$

Assuming that in the single fibril with orientation $\mathbf{n}$ the stress is $\sigma_{f}$ (the fibril stress averaged by fibril volume fraction), we calculate the global fibril stress $\tilde{\mathbf{T}}$, i.e. the homogenized single fibril stress relative to the global reference frame, through

$\tilde{\mathbf{T}}=\sigma_{f} \mathbf{n} \otimes \mathbf{n}$

The effective elastic stress tensor of the fibril network is given by

$\mathbf{T}^{f}=\int_{0}^{2 \pi} \int_{G_{1}(\phi)}^{H_{1}(\phi)} \tilde{\mathbf{T}} f(\phi, \theta) \sin \theta d \theta d \phi$

In order to calculate $\mathbf{T}^{f}$, we need to change the current variables $(\phi, \theta)$ to the initial variables $(\Phi, \Theta)$. Two effects influence the redistribution of fibrils [25]: the fibril initially oriented along $\mathbf{n}_{0}(\Phi, \Theta)$ rotates into $\mathbf{n}(\phi, \theta)$ and the differential area on a unit sphere, $d A_{0}=\sin \Theta d \Theta d \Phi$, changes into the new area $d A=\sin \theta d \theta d \phi$. Since the fibrils are considered to be material lines, fibril density over $d A_{0}$ will equal that over $d A$, so the mapping of the fibril distribution function under the deformation is given by

$f(\phi, \theta) \sin \theta d \theta d \phi=f_{0}(\Phi, \Theta) \sin \Theta d \Theta d \Phi$

Thus the effective fibril network stress can be calculated in terms of the initial fibril distribution function and the deformation gradient tensor, through equation (4),
$\mathbf{T}^{f}=\int_{0}^{2 \pi} \int_{G(\Phi)}^{H(\Phi)} \tilde{\mathbf{T}} f_{0}(\Phi, \Theta) \sin \Theta d \Theta d \Phi$

The lower and upper limits, $G(\Phi)$ and $H(\Phi)$, of the integral are obtained from the constraint inequality of tensile direction at a material point

$\varepsilon_{f}=\ln \left|\mathbf{F} \mathbf{n}_{0}\right|>0$

where we use the logarithmic strain measure to be compatible with the commercial finite element software ABAQUS, and the fibril stress has the form

$\tilde{\mathbf{T}}(R, \Phi, \Theta)=\frac{\sigma_{f}}{\left|\mathbf{F} \mathbf{n}_{0}\right|^{2}} \mathbf{F} \mathbf{n}_{0} \otimes \mathbf{F} \mathbf{n}_{0}$

The fibril network stress is added to the stress generated by the solid matrix and the stress in the fluid, to obtain the mixture Cauchy stress

$\mathbf{T}=-p \mathbf{I}+\frac{2}{J} \mathbf{F} \frac{\partial W}{\partial \mathbf{C}} \mathbf{F}^{T}+\int_{0}^{2 \pi} \int_{G(\Phi)}^{H(\Phi)} \tilde{\mathbf{T}} f_{0}(\Phi, \Theta) \sin \Theta d \Theta d \Phi(10)$

The hyperfoam strain energy function, which was proposed by Hill [36] and was subsequently employed to study the behavior of porous rubber [37], is used here to describe the behavior of the hyperelastic solid matrix

$W=\sum_{i=1}^{N} \frac{2 \mu}{\alpha_{i}^{2}}\left[\lambda_{1}^{\alpha_{i}}+\lambda_{2}^{\alpha_{i}}+\lambda_{3}^{\alpha_{i}}-3+\frac{1}{\beta}\left(J^{-\alpha_{i} \beta_{i}}-1\right)\right]$

For simplicity, we chose $N=1$, thus the hyperfoam potential of our model is given by

$W=\frac{2 \mu}{\alpha^{2}}\left[\lambda_{1}^{\alpha}+\lambda_{2}^{\alpha}+\lambda_{3}^{\alpha}-3+\frac{1}{\beta}\left(J^{-\alpha \beta}-1\right)\right]$

Here $\lambda_{1}, \lambda_{2}, \lambda_{3}$ are the principal stretches and $\mu, \alpha, \beta$ are material constants, where $\alpha$ and $\beta$ specify non-linearity of the matrix. The initial aggregate modulus $H_{A 0}$ and Poisson's ratio $v$ are related to the three constants by

$H_{A 0}=2(\beta+1) \mu$

$v=\frac{\beta}{1+2 \beta}$

The requirement for a stable material is $\mu>0$ and $\beta>-\frac{1}{3}$ and the upper limit of Poisson's ratio is 0.5 , which is the maximum Poisson's ratio of the non-fibrillar solid matrix. Other hyperelastic models can also be used to describe the behavior of non-fibrillar solid matrix. For example, the compressible neo-Hookean model was chosen by Wilson et al. [33]. The hyperfoam potential has advantages of using combinations of non-integer powers of stretches and separating distortion and dilatation in an attractive manner [37]. Moreover, it has been demonstrated to predict hyperelastic behavior of porous materials, consistent with the porous structure of cartilage, thus it might be a selectable constitutive model for articular cartilage.

The linear viscoelasticity of fibrils has been described by the 'standard solid model' [38], which is characterized by a spring in parallel with a Maxwell element. This model represents viscoelastic behavior in terms of three parameters: an effective elastic constant $E_{f}$, a viscosity $\eta_{f}$, and a relaxation 
time $\tau_{f}$. The stress-strain relation of the standard solid model is

$\sigma_{f}+\tau_{f} \dot{\sigma}_{f}=E_{f} \varepsilon_{f}+\left(\tau_{f} E_{f}+\eta_{f}\right) \dot{\varepsilon}_{f}$

where $\sigma_{f}$ and $\varepsilon_{f}$ represent one-dimensional stress and strain, respectively.

We wish to extend this linear viscoelastic model to include fibril nonlinearity. From the tensile stress-relaxation experiments [16, 31], it is observed that the characteristic relaxation time does not change significantly with strain, thus we may assume that the relaxation time $\tau_{f}$ of a fibril is constant. We also assume that the viscosity $\eta_{f}$ (a parameter of the dashpot in the viscoelastic model) of a fibril is independent of strain. However, the tangent Young's modulus of a fibril is assumed to be linearly related to the fibril strain inside the fibrils [14],

$E_{f}=E_{f_{0}}+E_{f_{1}} \varepsilon_{f}$

The corresponding secant modulus is given by

$\bar{E}_{f}=E_{f_{0}}+\frac{1}{2} E_{f_{1}} \varepsilon_{f}$

where $E_{f_{0}}$ and $E_{f_{1}}$ are curve-fitting parameters.

We construct the constitutive relationship for fibrils by substituting equation (17) into (15),

$\sigma_{f}+\tau_{f} \dot{\sigma}_{f}=\bar{E}_{f}\left(\varepsilon_{f}\right) \varepsilon_{f}+\bar{\eta}_{f}\left(\varepsilon_{f}\right) \dot{\varepsilon}_{f}$

where $\bar{\eta}_{f}\left(\varepsilon_{f}\right)=\tau_{f} \bar{E}_{f}\left(\varepsilon_{f}\right)+\eta_{f}$. The constitutive model, as seen in (18), is characterized by four material constants $E_{f_{0}}, E_{f_{1}}, \tau_{f}, \eta_{f}$

Darcy's law governs solid-fluid interaction, and the strain-dependent permeability proposed by Holmes and Mow [7] is employed. In terms of void ratio, permeability is given by

$k=k_{0}\left[\frac{\xi}{\xi_{0}}\right]^{\ell} \exp \left[\frac{M}{2}\left(\left(\frac{1+\xi}{1+\xi_{0}}\right)^{2}-1\right)\right]$

where $\xi=\phi^{w} /\left(\phi^{s}+\phi^{f}\right)$ represents void ratio, $k$ is the permeability, $M$ is a constant, and the subscript zero indicates initial values. We assume constant $\ell=0.0848$ [7] and specify $\xi_{0}=4.0[39,40]$. by

At equilibrium, the fibril-network stress tensor is given

$\mathbf{T}^{f}=\int_{0}^{2 \pi} \int_{G(\Phi)}^{H(\Phi)} \mathbf{n} \otimes \mathbf{n} \bar{E}_{f}\left(\varepsilon_{f}\right) \varepsilon_{f} f_{0}(\Phi, \Theta) \sin \Theta d \Theta d \Phi$

and the three principal stresses of the composite under uniaxial mode are

$t_{1}=T_{11}^{f}+\frac{2}{J} \frac{\mu}{\alpha}\left(\lambda_{1}^{\alpha}-J^{-\alpha \beta}\right)$

$t_{2}=T_{22}^{f}+\frac{2}{J} \frac{\mu}{\alpha}\left(\lambda_{2}^{\alpha}-J^{-\alpha \beta}\right)$

$t_{3}=T_{33}^{f}+\frac{2}{J} \frac{\mu}{\alpha}\left(\lambda_{3}^{\alpha}-J^{-\alpha \beta}\right)$

To show the capability of our microstructural model, we consider the articular cartilage as consisting of three zones, with fibril orientation changing from zone to zone. Relative zonal thicknesses are assumed as $15 \%$ for the surface, $55 \%$ for the middle and $30 \%$ for the deep zone [1]. Fibril orientations in the different zones are suggested by microscopic examinations [41-43], with distribution functions as given by Lei and Szeri [17].

The middle zone contains initially random fibrils

$f_{R}(\Phi, \Theta)=\frac{1}{4 \pi}$ by

In the deep zone, initial fibril distribution is characterized

$$
f_{V}(\Phi, \Theta)=\frac{e^{-\frac{\Theta^{2}}{0.5}}+e^{-\frac{(\pi-\Theta)^{2}}{0.5}}}{2 \pi \int_{0}^{\pi}\left[e^{-\frac{\alpha^{2}}{0.5}}+e^{-\frac{(\pi-\alpha)^{2}}{0.5}}\right] \sin \alpha d \alpha}
$$

In the surface zone, the fibrils are initially distributed according to

$$
f_{H}(\Phi, \Theta)=\frac{e^{-\frac{(\Theta-\pi / 2)^{2}}{0.5}}}{2 \pi \int_{0}^{\pi} e^{-\frac{(\alpha-\pi / 2)^{2}}{0.5}} \sin \alpha d \alpha}
$$

For simplicity, we assume that both the properties of the fibril and the properties of the solid matrix are identical in the three zones, though this assumption is not crucial. Note that the fibril distribution functions used here are only a trial geometric configuration that is intended to mimic fibril organization in the three zones but may not be a true representation of any actual structure [17].

\subsection{Finite Element Implementation}

We implement the nonlinear microstructural poroelastic model in the commercial finite element software ABAQUS, employing the SOIL consolidation procedure. The 4-node axisymmetric porous element (CAX4P) is employed for the solid matrix, and its mechanical behavior is specified by the HYPERFOAM option. The 4-node axisymmetric solid continuum element (CAX4), which shares the identical nodes with the porous element, is adopted for the fibril network. Material behavior of the fibril element is specified by a userdefined subroutine UMAT. In this subroutine, we update the stresses according to the deformation gradient at each increment.

Applying central difference operator to equation (18), we obtain the stress increment of a fibril in the time inter$\operatorname{val}(t, t+\Delta t)$

$\Delta \sigma_{f}=\frac{\bar{E}_{f}\left(\varepsilon_{f}\right) \Delta t / 2+\bar{\eta}_{f}\left(\varepsilon_{f}\right)}{\Delta t / 2+\tau_{f}} \Delta \varepsilon_{f}+\frac{\bar{E}_{f}\left(\varepsilon_{f}\right) \Delta t}{\Delta t / 2+\tau_{f}} \varepsilon_{f}-\frac{\Delta t}{\Delta t / 2+\tau_{f}} \sigma_{f}$

where $\varepsilon_{f}=\varepsilon_{f}(t)$ and $\sigma_{f}=\sigma_{f}(t)$ are (one dimensional) fibril strain and stress, respectively, at time $t$.

In order to calculate the fibril network stress, we need to calculate and sum individual fibril stress at current time. The present study approximates global fibril network stresses as follows. Assuming to have available both fibril strain and fibril stress at time $\mathrm{t}$, we begin computing the increments to these quantities during the time step $\Delta t$. The strain increments $\Delta \mathbf{e}$ are obtained from the virtual work principle in 
ABAQUS where the virtual field satisfies the boundary conditions of the problem.

Rotating the individual fibril stress increment, equation (27), into the global reference frame we have

$$
\Delta \tilde{\boldsymbol{T}}=\Delta \sigma_{f} \boldsymbol{n} \otimes \boldsymbol{n}+\frac{2 \sigma_{f} \boldsymbol{n}}{\sqrt{\boldsymbol{n}_{0} \cdot \boldsymbol{C} \boldsymbol{n}_{0}}} \otimes\left[\Delta \boldsymbol{F} \boldsymbol{n}_{0}+\left(\boldsymbol{n}_{0} \cdot \Delta \boldsymbol{C} \boldsymbol{n}_{0}\right) \boldsymbol{n}\right]
$$

However, the finite element implementation of Eq. (28a) is too complex and we approximate it by neglecting the second term due to change of fiber orientation because of altered strain. Thus, in place of Eq. (28a) we employ

$$
\Delta \tilde{\mathbf{T}} \approx\left[\frac{\bar{E}_{f}\left(\varepsilon_{f}\right) \Delta t / 2+\bar{\eta}_{f}\left(\varepsilon_{f}\right)}{\Delta t / 2+\tau_{f}} \Delta \varepsilon_{f}+\frac{\bar{E}_{f}\left(\varepsilon_{f}\right) \Delta t}{\Delta t / 2+\tau_{f}} \varepsilon_{f}-\frac{\Delta t}{\Delta t / 2+\tau_{f}} \sigma_{f}\right] \mathbf{n} \otimes \mathbf{n}
$$

in the finite element representation. The stiffness matrix may be affected by the approximation. However, the error introduced by the approximation (28b) is not significant when the loading strain is small; this can be shown by comparison of equilibrium stress calculated from finite element simulation and from FORTRAN code. work

Integrating, yields the stress increment of the fibril net-

$$
\begin{aligned}
\Delta \mathbf{T}^{f}(\mathrm{t}) & =\int_{0}^{2 \pi} \int_{G(\Phi)}^{H(\Phi)} \Delta \tilde{\mathbf{T}} f_{0}(\Phi, \Theta) \sin \Theta d \Theta d \Phi \\
& \approx \mathbf{B} \Delta \mathbf{e}+\mathbf{D} \mathbf{e}(t)-\frac{\Delta t}{\Delta t / 2+\tau_{f}} \mathbf{T}^{f}(\mathrm{t})
\end{aligned}
$$

Here

$$
\begin{gathered}
B_{i j k l}=\int_{0}^{2 \pi} \int_{G(\Phi)}^{H(\Phi)} \frac{\bar{E}_{f}\left(\varepsilon_{f}\right) \Delta t / 2+\bar{\eta}_{f}\left(\varepsilon_{f}\right)}{\Delta t / 2+\tau_{f}} n_{i} n_{j} n_{k} n_{l} f_{0}(\Phi, \Theta) \sin \Theta d \Theta d \Phi \\
\mathrm{D}_{i j k l}=\int_{0}^{2 \pi} \int_{G(\Phi)}^{H(\Phi)} \frac{\bar{E}_{f}\left(\varepsilon_{f}\right) \Delta t}{\Delta t / 2+\tau_{f}} n_{i} n_{j} n_{k} n_{l} f_{0}(\Phi, \Theta) \sin \Theta d \Theta d \Phi
\end{gathered}
$$

Equation (29) shows that the increment in fibril stress $\Delta \mathbf{T}^{f}$ depends on the current strain $\mathbf{e}(t)$, the current stress $\mathbf{T}^{f}(t)$, and the strain increment $\Delta \mathbf{e}$, as well as the step size $\Delta t$. Had we used the integral representation of stress, we could show that its increment depends on the history of strain, not only its current value. Having computed the stress increment $\Delta \mathbf{T}^{f}$, we calculate the fibril-network stress tensor at time $t+\Delta t$ as

$\mathbf{T}^{f}(\mathrm{t}+\Delta \mathrm{t})=\mathbf{T}^{f}(\mathrm{t})+\Delta \mathbf{T}^{f}$

\subsection{Computational Cases}

\section{Equilibrium Strain-Dependent Behavior: Single Tissue Layer}

We investigate the equilibrium stress-strain behavior of this model in uniaxial tension, on a cubic sample from different zones with the loading direction (1-direction) parallel to cartilage surface. The fibril orientations for the three different zones are described by equations (24-26). The boundary conditions are that the stretch $\lambda_{1}$ is known, and $t_{2}=t_{3}=0$; therefore, we need to solve equations (22-23) to get the other two stretches $\lambda_{2}, \lambda_{3}$, and to calculate stress $t_{1}$ using equation (21). These equations are solved using computational methods via FORTRAN codes. From the calculated stretch $\lambda_{2}$, we can compute the apparent Poisson's ratio $v_{12}=\left(1-\lambda_{2}\right) /\left(\lambda_{1}-1\right)$ as a function of nominal tensile strain.
Time-Dependent Behavior: Composite (Three-Layered) Tissue

The sensitivity of the constitutive model to timedependent behavior is investigated before the model is fitted to the experimental data. Then two groups of experimental data are chosen to validate the proposed model. Firstly, the model is fitted to published experimental stress-relaxation data in both tension and unconfined compression [16]. Secondly, we fit to time-dependent load and lateral displacement data in unconfined compression [26], and predict stressrelaxation behavior in indentation test. Consequently, we demonstrate that the model, using a unique set of parameters per cartilage sample, can predict the outcome of three different tests. We employ the relative error as a criterion for assessing goodness of fit. An optimization procedure [44] that combines ABAQUS and optimization tools in MATLAB is employed to curve-fit experimental data.

The cartilage sample consists of three zones, with $15 \%$, $55 \%, 30 \%$ in relative thickness for surface, middle, and deep zones, respectively. Axisymmetric finite element meshes are employed for simulations. The specimen-platen contact is assumed to be adhesive in the tests of Li et al. [16], and the boundary conditions for unconfined compression and tension are given by

$$
\begin{array}{lrl}
u_{z}=u(t), u_{r}=0, U_{z}=u_{z} & \text { at } z=h, \\
u_{z}=0, u_{r}=0, U_{z}=u_{z} & \text { at } z=0, \\
u_{r}=0, U_{r}=0 & \text { at } r=0, \\
p=0 & \text { at } r=R .
\end{array}
$$

where $\left(u_{r}, u_{z}\right)$ and $\left(U_{r}, U_{z}\right)$ are components of the displacement vectors of the solid phase and fluid phase, respectively, and $p$ is the pore pressure.

For unconfined compression tests of Disilvestro and Suh [26], the specimen-platen contact is assumed to be frictionless. The boundary conditions are similar to equation (33) except that $u_{r}$ is not constrained at $z=h$ and $z=0$. Since the lateral displacement is not uniform throughout the thickness, we use the averaged lateral displacement in the curvefitting.

The following boundary conditions are specified for indentation test using porous indenter: the nodes are confined in all directions at $z=0$; the nodes are confined in radial direction at $r=0$; the nodes are constraint by zero pore pressure at $r=R$ and at $z=h$; the rigid porous indenter is compressed by applying specified axial displacement $u(t)$; the contact between cartilage specimen and indenter is frictionless. The axial load is computed from the top surface of the indenter.

\section{RESULTS}

\subsection{Equilibrium Strain-Dependent Behavior: Single Tis- sue Layer}

We investigate the effect of fibril nonlinearity on the stress and the lateral Poisson's ratio $v_{12}$. Fig. (1) shows equilibrium stress and lateral Poisson's ratio varying with nominal strain in tension for random fibril distribution, when we fix parameter values at $H_{A 0}=0.6 \mathrm{MPa}, v=0.4, \alpha=1.0$, $E_{f_{0}}=30 \mathrm{MPa}$, but vary the ratio of $E_{f_{1}} / E_{f_{0}}$. It may be observed that both stress and lateral Poisson's ratio curves shift 
upwards as $E_{f_{1}} / E_{f_{0}}$ is increased. Apparently, the nominal stress-strain curves have a toe region and a linear region (Fig. 1A), which is consistent with experimental observations [32]. Though the tangent fibril stiffness is straindependent, the nominal stress may be reduced due to the significant transverse contraction. The lateral Poisson's ratio does not change significantly with nominal strain when the ratio of $E_{f_{1}} / E_{f_{0}}$ is fixed (Fig. 1B), but it is greater than 0.5 , indicating significant fibril-induced anisotropy. For surface and deep zones, similar strain-dependent results are calculated, though tensile modulus and lateral Poisson's ratio are higher in surface zone and lower in deep zone than that in middle zone.

(A)

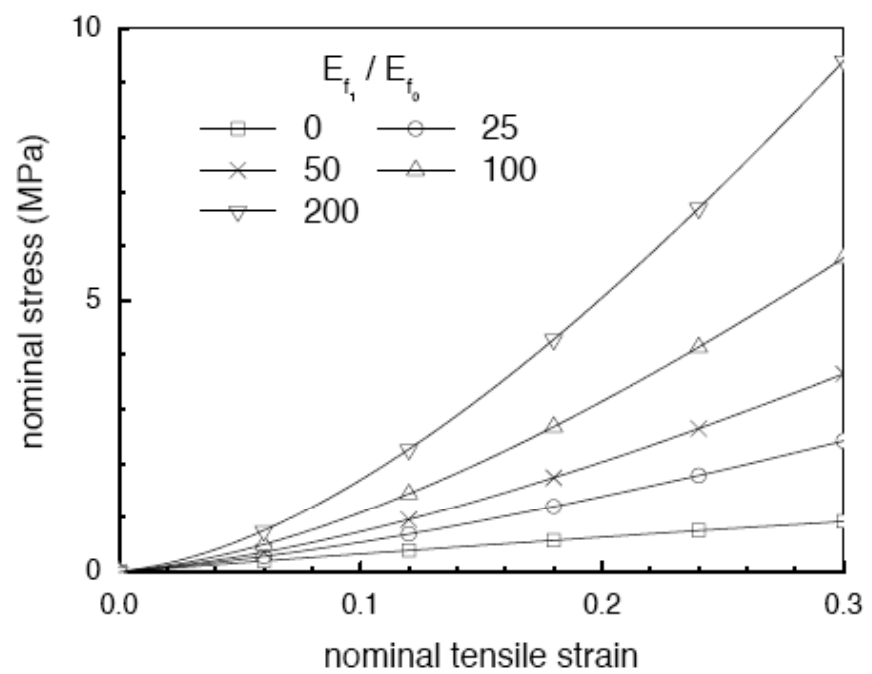

(B)

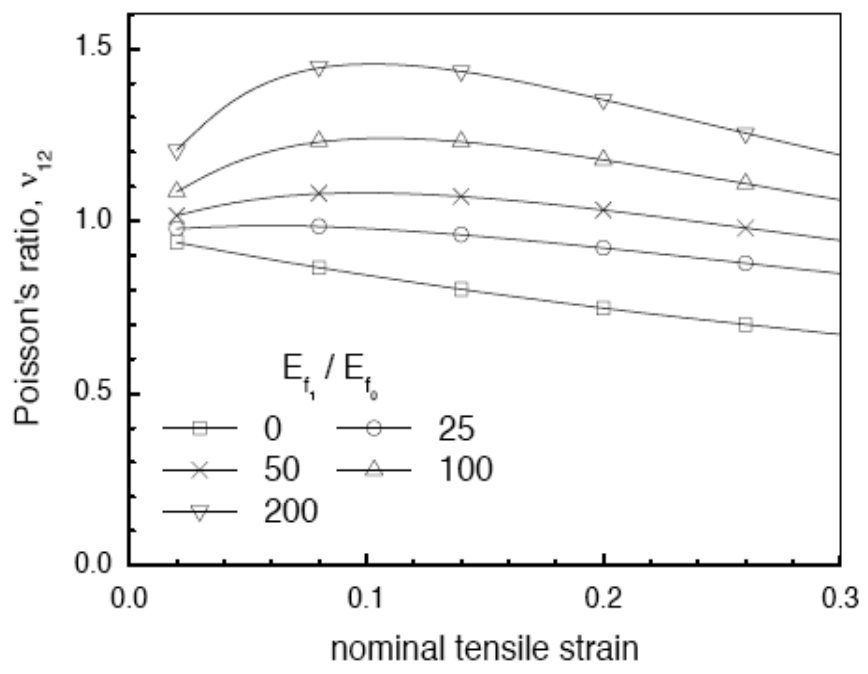

Fig. (1). Effects of fibril nonlinearity on the equilibrium stressstrain responses in tension for random fibril distribution: (A) nominal stress; (B) lateral Poisson's ratio. The parameter values are fixed at $H_{A 0}=0.6 \mathrm{MPa}, v=0.4, \alpha=1.0, E_{f_{0}}=30 \mathrm{MPa}$, while the ratio $E_{f_{1}} / E_{f_{0}}$ changes.

Huang et al. [31] found that the Poisson's ratio was approximately constant within the range of 6-12\% tensile strain from their experiments. Elliott et al. [32] also found that the
Poisson's ratio was almost independent of strain up to $15 \%$ tensile strain. These experimental findings are consistent with our model predictions. Furthermore, their measured values of lateral Poisson's ratio are within the range of our computational results.

In previous study of a linear microstructural model, we found that the tensile Poisson's ratio was mainly determined by $E_{f} / H_{A}$ ratio [17]. However, in the present nonlinear microstructural model, both $E_{f}$ and $H_{A}$ change with strain. A fibril that has smaller incline angle to the load will undertake larger tensile strain, which makes effective fibril stiffness change with direction. All these factors contribute to the tensile Poisson's ratio, and make the behavior of straindependent Poison's ratio not easy to forecast. As shown in Fig. (1), the tensile Poisson's ratio may increase first then decrease later as the strain increases, or it may be strainindependent.

\subsection{Time-Dependent Behavior: Composite (Three- Layered) Tissue}

\section{Sensitivity Analysis}

The sensitivity is investigated on 9 parameters in both unconfined compression and tension stress-relaxation using a calculated target. The target stress-relaxation is computed based on the target parameters,

$$
\begin{aligned}
& H_{A 0}=0.8 \mathrm{MPa}, v=0.4, \alpha=0.5, k_{0}=3.0 \times 10^{-16} \mathrm{~m}^{4} \mathrm{~N}^{-1} s^{-1}, \\
& M=6.0, \tau_{f}=120 s, \\
& E_{f_{0}}=10.0 \mathrm{MPa}, E_{f_{1}}=2500 \mathrm{MPa}, \\
& \eta_{f}=1.5 \times 10^{4} \mathrm{MPa} \mathrm{s} .
\end{aligned}
$$

The sample dimension are $\mathrm{R}=1.5 \mathrm{~mm}, \mathrm{H}=1.0 \mathrm{~mm}$. There are 5 stress-relaxation steps; one-step time is 500 seconds, and the loading rate is $1 \%$ in 10 seconds for both unconfined compression and tension. The sensitivity analysis is performed by changing each parameter in turn by $\pm 20 \%$, while keeping all other parameters fixed at their target values. It is found that the Poisson's ratio of solid matrix $v$ is the most sensitive parameter, while $\alpha$ and $M$ are the least sensitive parameters. The computational stress-relaxation curves in unconfined compression and tension by changing parameter $\eta_{f}$ are shown in Fig. (2).

To indicate the part of the time-dependence due to the biphasic nature of the cartilage through Darcy's law and the part due to the purely viscoelastic behavior of the fibrils, we present a comparison among fluid pressurization, purely elastic and viscoelastic fibrils on the time-dependence of the material response. The target curves are calculated using the same parameters and loading rates as those in Fig. (2). For a clearer view, only one single loading-unloading ramp but with different fibril moduli ( $E_{f_{0}}=10 \mathrm{MPa}$ and $E_{f_{0}}=100 \mathrm{MPa}$ ) is shown in Fig. (3), where the purely elastic fibril network is represented by $\eta_{f}=0$ and $\tau_{f}=0$; the timedependence of the material response due to purely viscoelastic behavior of the fibril network is specified by no fluid pressurization $(k \rightarrow \infty)$.

It is observed that fluid pressurization and fibril viscoelasticity play important roles on the time-dependent behavior 
(A)

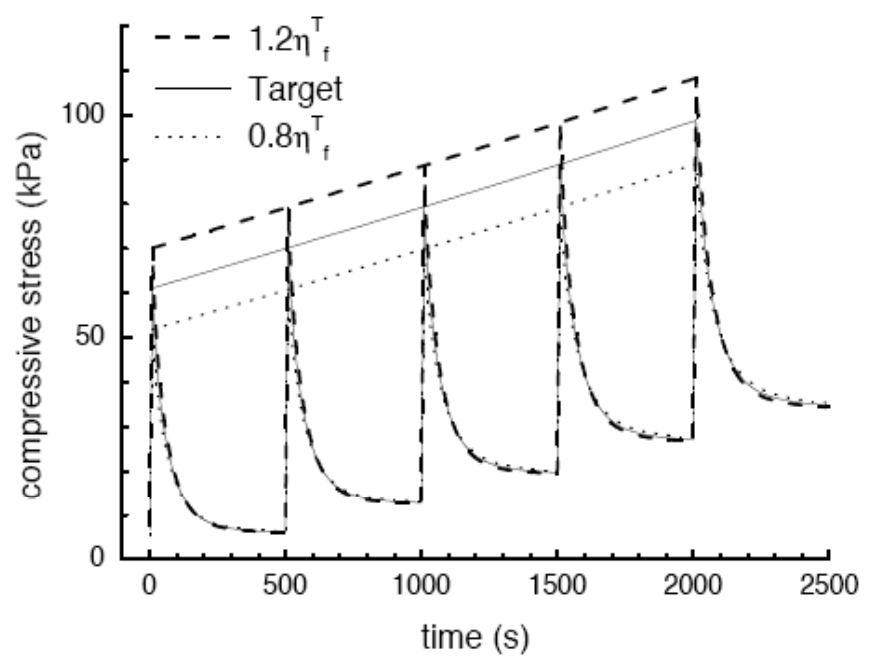

(B)

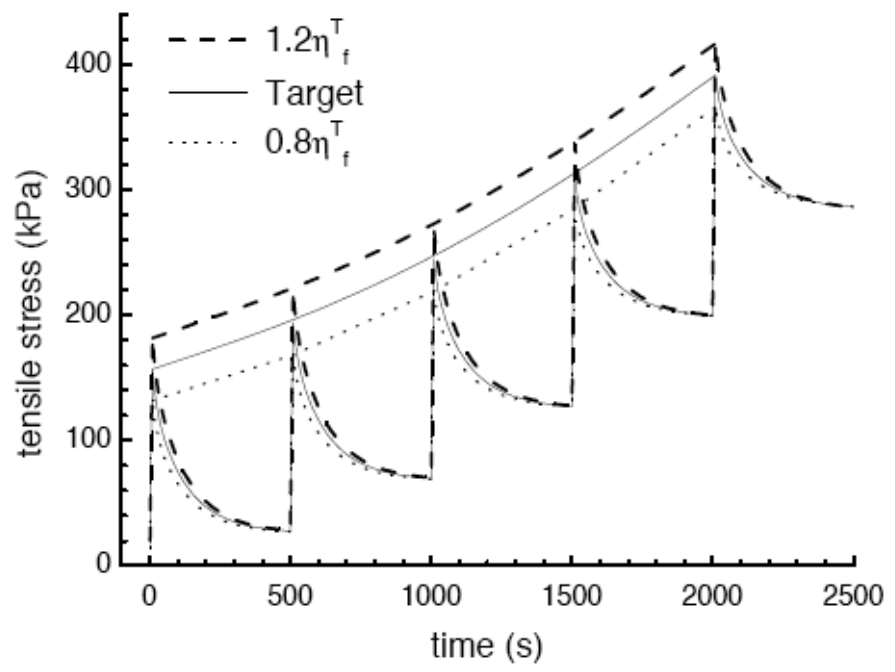

Fig. (2). Sensitivity of stress-relaxation curve to the changes in $\eta_{f}$ : (A) unconfined compression; (B) tension.

of the tissue in both unconfined compression and tension (Fig. 3). For unconfined compression, if there is no fluid pressurization, stress-relaxation is not observed; if fibrils are purely elastic, the peak stress is decreased significantly, though the extent of reduction is dependent on the fibril modulus (Fig. 3A). For uniaxial tension, if fibrils are purely elastic, the stress-relaxation behavior is still observed, which is due to the fluid pressurization; if there is no fluid pressurization, the peak-to-equilibrium stress ratio is much lower (Fig. 3B). Because of the large transverse contraction during tensile loading, the interstitial fluid is pressurized. The pore pressure will resist transverse contraction and tighten more fibrils, therefore enhance the stress-relaxation in uniaxial tension.

\section{Unconfined Compression and Tension}

Li et al. [16] performed tensile and compressive stressrelaxation tests in the axial direction, where cartilage samples were glued between loading platens. We take their published experimental data and fit them with our model. The dimension of selected cartilage sample is $R=1.82 \mathrm{~mm}$ and $h=1.13 \mathrm{~mm}$, and each step loading is $0.6 \%$ strain.
(A)

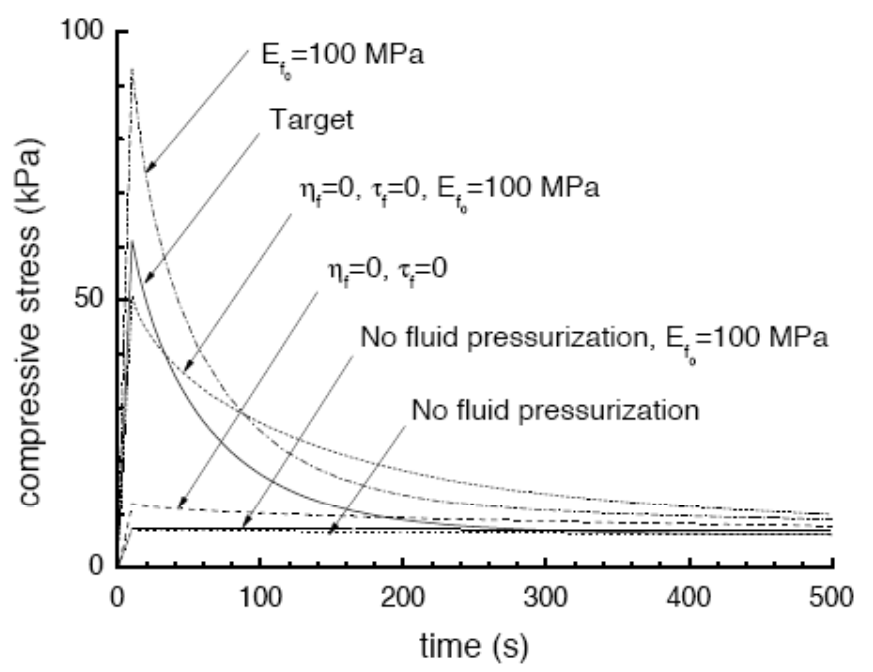

(B)

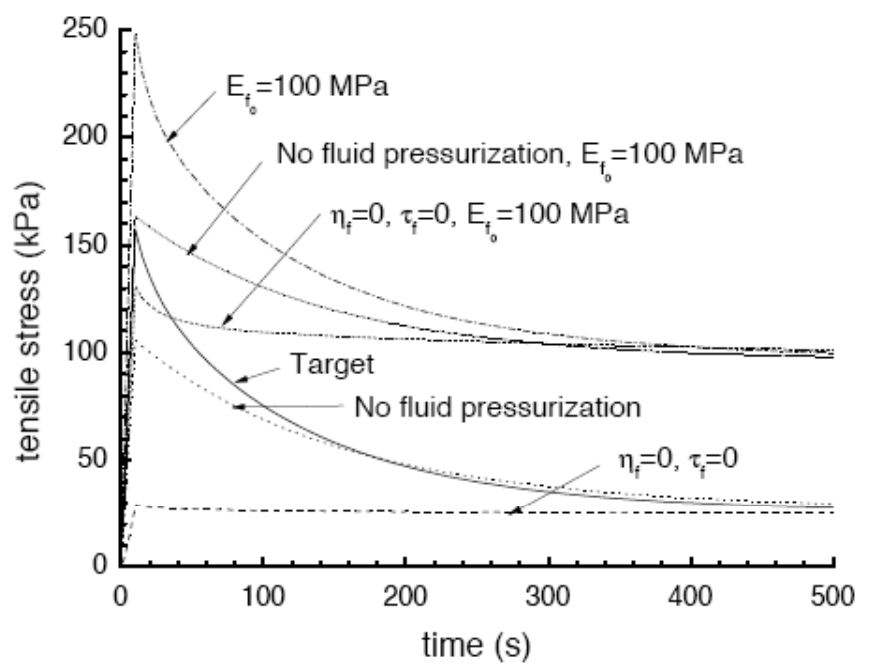

Fig. (3). Effects of fluid pressurization and fiber viscoelasticity on the time-dependent response: (A) unconfined compression; (B) tension. Curves not labeled for $\mathrm{E}_{\mathrm{f}_{0}}$ have $\mathrm{E}_{\mathrm{f}_{0}}=10 \mathrm{MPa}$.

The model parameter values are obtained by curve-fitting simultaneously experimental stress-relaxation data in unconfined compression and tension tests.

$H_{A 0}=1.64 \mathrm{MPa}, v=0.361$,

$\alpha=0.16, k_{0}=7.05 \times 10^{-17} \mathrm{~m}^{4} \mathrm{~N}^{-1} s^{-1}$,

$M=4.8, \tau_{f}=102 \mathrm{~s}$,

$E_{f_{0}}=105 \mathrm{MPa}, E_{f_{1}}=5670 \mathrm{MPa}$,

$\eta_{f}=2.21 \times 10^{4}$ MPa s.

Figs. (4,5) show experimental and predicted stressrelaxation curves in tension $\left(R^{2}=0.992\right)$, and in unconfined compression $\left(R^{2}=0.983\right)$, respectively. Agreement between model prediction and experiment is satisfactory. Therefore, our model shows the capability to fit stress-relaxation in both axial tension and unconfined compression on an identical cartilage sample.

In agreement with findings of $\mathrm{Li}$ et al. (2005), it is observed that the aggregated modulus for the solid matrix is higher than those reported in the literature. This difference 
may be partially due to that the stress-relaxation does not reach equilibrium in unconfined compression (Fig. 5). It may also due to different cartilage source, and experimental method using glue between sample and platen. To obtain more detailed material properties of articular cartilage, we need various experimental data in future studies that will highlight the effects of large deformation.

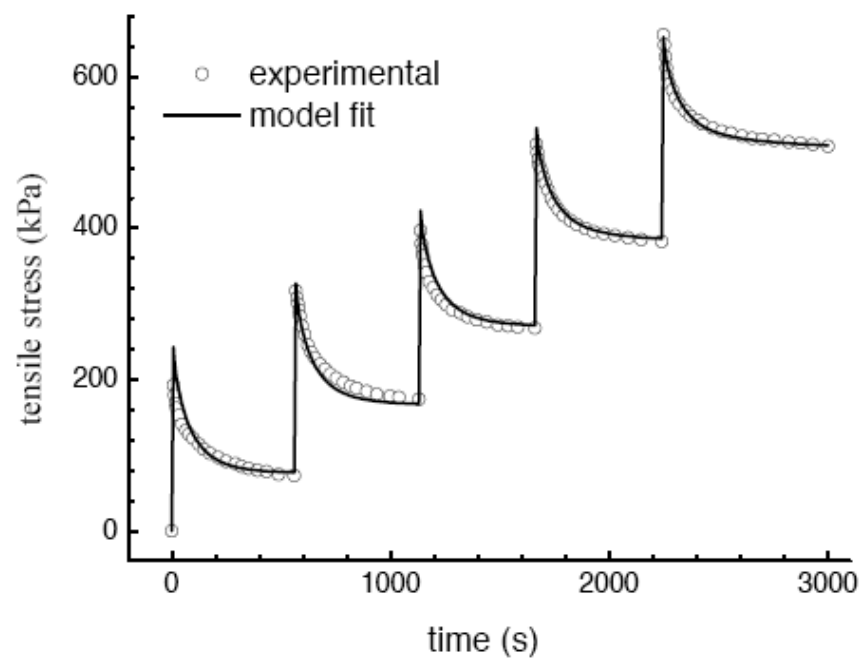

Fig. (4). Curve-fit to stress-relaxation data in tension (data, [16]).

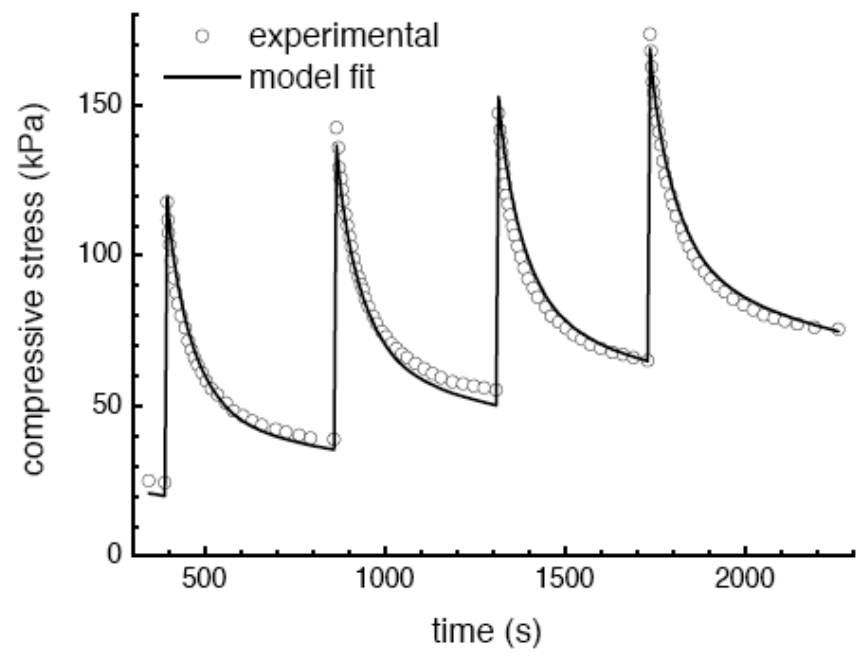

Fig. (5). Curve-fit to stress-relaxation data in unconfined compression (data, [16]).

\section{Unconfined Compression and Indentation}

Disilvestro and Suh [26] performed unconfined compression on eighteen cartilage plugs $(h=1.28 \mathrm{~mm}, R=1.5 \mathrm{~mm})$ from 1-2 years old bovine. They measured both axial reaction force and lateral displacement changing with time. Additionally, four of the eighteen specimens were tested in an indentation stress-relaxation protocol using a porous indenter $\left(r_{\text {ind }}=1.01 \mathrm{~mm}\right)$. Both unconfined compression and indentation tests consist of a preload of $10 \%$ strain, equilibration for 15 minutes, and a ramp test of $5 \%$ strain at a rate of $0.1 \%$ strain/s. We take these two kinds of experimental data and compare with our model predictions.

The model parameter values are obtained by fitting to the measured axial load and lateral displacement simultaneously in unconfined compression.
$H_{A 0}=0.57 \mathrm{MPa}, v=0.4, \alpha=0.15$,

$k_{0}=1.26 \times 10^{-15} \mathrm{~m}^{4} \mathrm{~N}^{-1} \mathrm{~s}^{-1}, M=11.2, \tau_{f}=226 \mathrm{~s}$

$E_{f_{0}}=12.1 \mathrm{MPa}, E_{f_{1}}=5017 \mathrm{MPa}$,

$\eta_{f}=3.24 \times 10^{4}$ MPa s.

The experimental and predicted axial loads (normalized to equilibrium load) are shown in Fig. (6A), indicating good agreement $\left(R^{2}=0.995\right)$. Fig. (6B) shows that experimental and predicted lateral displacements also agree well $\left(R^{2}=0.936\right)$. We conclude that the constitutive model predicts successfully both time-dependent axial reaction force and lateral displacement in unconfined compression. The values for aggregate modulus and permeability agree well with those reported in the literature [15, 26].

(A)
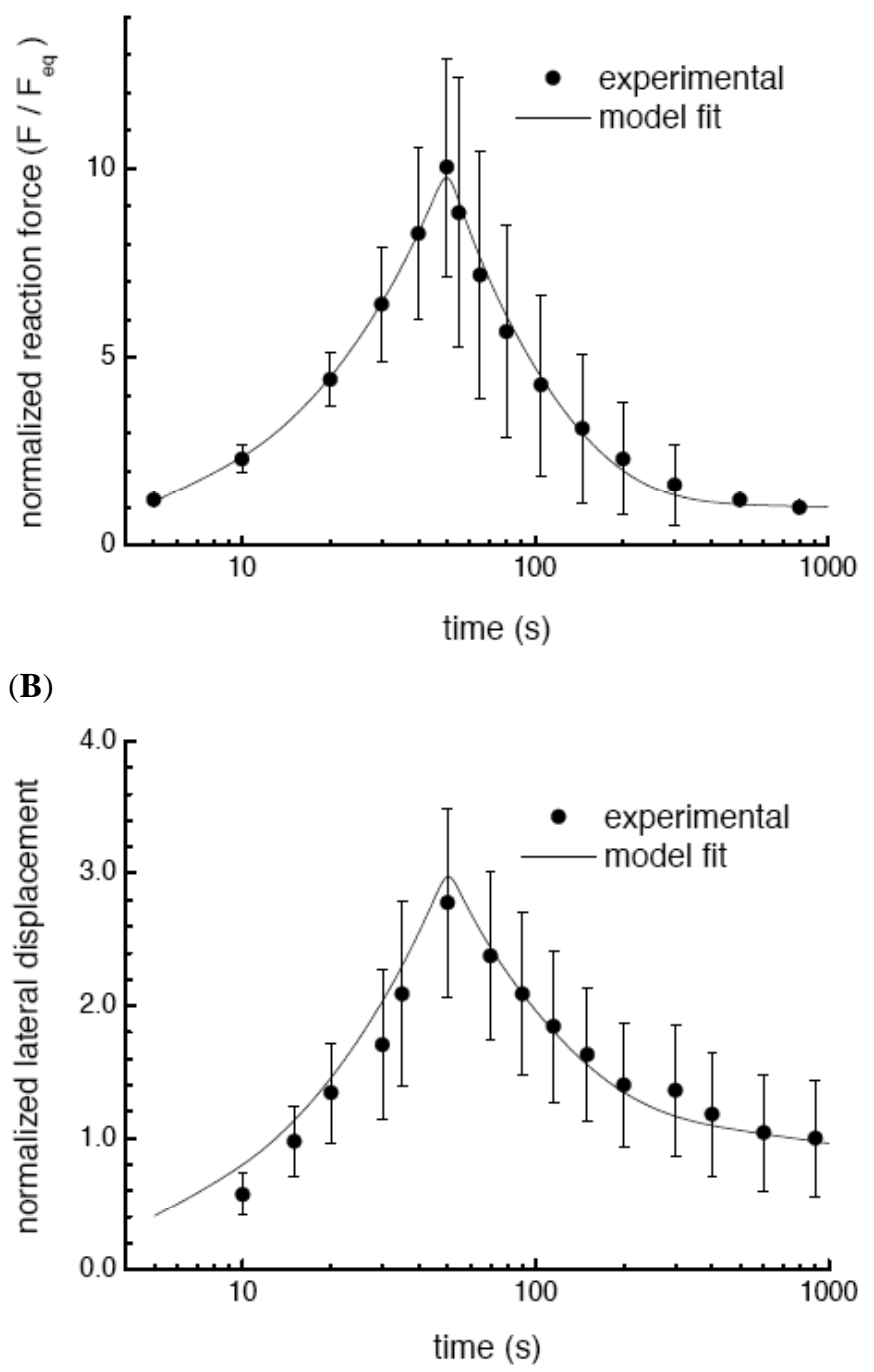

Fig. (6). Curve-fits to data of Disilvestro and Suh [26] in unconfined compression: (A) normalized axial load; (B) normalized lateral displacement.

Using the parameter values obtained from unconfined compression, we predict the axial load and compare it with experimental data in indentation test using a porous indenter. The thickness of the specimen is $1.28 \mathrm{~mm}$, and the radius of the indenter is $1.01 \mathrm{~mm}$, while the radius of the specimen is assumed to be four times of the radius of the indenter. Fig. 
(7) shows experimental and predicted axial loads (normalized to equilibrium load) in the indentation test $\left(R^{2}=0.94\right)$. It is observed that the model predicts a somewhat higher load during the relaxation period. Since only four of the eighteen specimens are tested in indentation, there is a difference in population between unconfined compression and indentation. That may partially account for the mismatch between experimental data and model prediction in indentation using the parameter values obtained from unconfined compression curve-fits. Nevertheless, our model shows the potential to simulate time-dependent mechanical behavior in indentation.

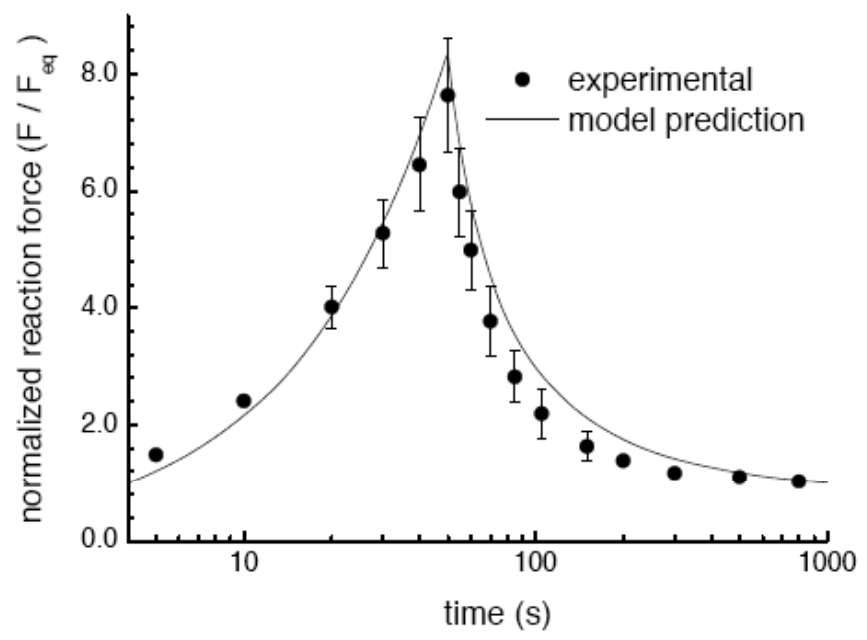

Fig. (7). Comparison of normalized axial load from indentation test [26] with model prediction.

\section{DISCUSSION}

To overcome the limitations of small deformation models, a nonlinear microstructural model is developed. This model can be applied to large deformation, incorporate arbitrary fibril distribution, fibril nonlinearity and fibril viscoelasticity, though we have employed a specific fibril stressstrain relation and a three-layered fibril configuration in the present study. A finite element method is presented for the microstructural model.

The fibril distribution functions for three zones are identical to that in a previous study [17]. A nonlinear viscoelastic constitutive model based on standard solid model is proposed to mimic fibril behavior. The fibril stress-strain relation is different from that of Li et al. [16] and that of Wilson et al. [15], but all these previous models have already shown the effects of fibril viscoelasticity and demonstrated capability to fit to experimental data to some extent. Though in this study we do not incorporate inhomogeneity and swelling effects of solid matrix, such effects can be incorporated into our model in the manner of Wilson et al. [33]. The thrust behind our model development is to minimize the number of model parameters, while at the same time to capture the behavior of the tissue.

Based on specific fibril structure and constitutive law, a new set of material parameters are defined. An analytical method is developed to study strain-dependent behavior at equilibrium in tension and unconfined compression. The whole set of parameter values can be determined by curvefitting time-dependent experimental data. Our model can predict strain-dependent equilibrium stiffness and Poisson's ratio of cartilage in tension. The model can also simulate stress-relaxation behavior of cartilage in tension, unconfined compression, and indentation, and time-dependent lateral displacement in unconfined compression.

\section{ACKNOWLEDGEMENTS}

This publication was made possible by Grant Number P20RR16458 from the National Center for Research Resources (NCRR), a component of the National Institutes of Health (NIH). Its contents are solely the responsibility of the authors and do not necessarily represent the official views of NCRR or NIH.

\section{REFERENCES}

[1] Mow VC, Ratcliffe A, Poole AR. Cartilage and diarthrodial joints as paradigms for hierarchical materials and structures. Biomaterials 1992; 13: 67-97.

[2] Mow VC, Lai WM. Recent developments in synovial joint biomechanics. SIAM Review 1980; 22: 275-317.

[3] Zhang L, Szeri, AZ. Transport of neutral solute in articular cartilage: effects of loading and particle size. Proc R Soc Lond A 2005 461: 2021-42

[4] Hayes WC, Keer, LM, Herrmann, G, Mockros, LF. A mathematical analysis for indentation tests of articular cartilage. J Biomech 1972; 5: 541-51.

[5] Mow VC, Kuei SC, Lai WM, Armstrong CG. Biphasic creep and stress relaxation of articular cartilage in compression: Theory and Experiments. J Biomech Eng 1980; 102: 73-84.

[6] Mak AF. The apparent viscoelastic behavior of articular cartilage - The contributions from the intrinsic matrix viscoelasticity and interstitial fluid flows. J Biomech Eng 1986; 108: 123-30.

[7] Holmes MH, Mow VC. The nonlinear characteristics of soft gels and hydrated connective tissues in ultrafiltration. J Biomech 1990; 23: $1145-56$.

[8] Lai WM, Hou JS, Mow VC. A triphasic theory for the swelling and deformation behaviors of articular cartilage. J Biomech Eng 1991; 113: $245-58$

[9] Oloyede A, Broom, ND. Is classical consolidation theory applicable to articular cartilage deformation? Clin Biomech 1991; 6: 20612 .

[10] Soulhat J, Buschmann MD, Shirazi-Adl, A. A fibril-networkreinforced biphasic model of cartilage in unconfined compression. J Biomech Eng 1999; 121: 340-7.

[11] Cohen B, Lai, WM, Mow VC. A transversely isotropic biphasic model for unconfined compression of growth plate and chondroepiphysis. J Biomech Eng 1998; 120: 491-6.

[12] Soltz MA, Ateshian GA. A conewise linear elasticity mixture model for the analysis of tension-compression nonlinearity in articular cartilage. J Biomech Eng 2000; 122: 576-86.

[13] Nguyen T, Oloyede A. Predictive rheological models for the consolidation behaviour of articular cartilage under static loading. Proc Instn Mech Engrs Part H: J. Eng Medicine 2001; 215: 565-77.

[14] Li LP, Soulhat J, Buschmann MD, Shirazi-Adl A. Nonlinear analysis of cartilage in unconfined ramp compression using a fibril reinforced poroelastic model. Clin Biomech 1999; 14: 673-82.

[15] Wilson W, van Donkelaar CC, van Rietbergen B, Ito K, Huiskes R. Stresses in the local collagen network of articular cartilage: a poroviscoelastic fibril-reinforced finite element study. J Biomech 2004; 37: 357-66.

[16] Li LP, Herzog W, Korhonen RK, Jurvelin JS. The role of viscoelasticity of collagen fibers in articular cartilage: axial tension versus compression. Med Eng Physics 2005; 27: 51-7.

[17] Lei FL, Szeri AZ. The influence of fibril organization on the mechanical behavior of articular cartilage. Proc R Soc Lond A 2006; 462: 3301-22.

[18] Hill R. Theory of mechanical properties of fibre-strengthened materials: I. elastic behaviour. J Mech Phys Solids 1964; 12: 199-212.

[19] Christensen RM, Waals FM. Effective stiffness of randomly oriented fibre composites. J Comp Materials 1972; 6: 518-32.

[20] Advani SG, Tucker III CL. The use of tensors to describe and predict fiber orientation in short fiber composites. J Rheology 1987; 31: 751-84. 
[21] Chou TW. Microstructural Design of Fiber Composites. Cambridge University Press: Cambridge; 1992.

[22] Aspden RM. Relation between structure and mechanical behavior of fibre-reinforced composite materials at large strains. Proc R Soc Lond A 1986; 406: 287-98.

[23] Farquhar T, Dawson PR, Torzilli PA. A microstructural model for the anisotropic drained stiffness of articular cartilage. J Biomech Eng 1990; 112: 414-25.

[24] Ault HK, Hoffman AH. A composite micromechanical model for connective tissues: Part I — theory. J Biomech Eng 1992; 114: $137-41$.

[25] Schwartz MH, Leo PH, Lewis JL. A microstructural model for the elastic response of articular cartilage. J Biomech 1994; 27: 865-73.

[26] DiSilvestro MR, Suh JKF. A cross-validation of the biphasic poroviscoelastic model of articular cartilage in unconfined compression, indentation, and confined compression. J Biomech 2001; 34: 51925 .

[27] Huang CY, Soltz MA, Kopacz M, Mow VC, Ateshian GA. Experimental verification of the roles of intrinsic matrix viscoelasticity and tension-compression nonlinearity in the biphasic response of cartilage. J Biomech Eng 2003; 125: 84-93.

[28] Bursac PM, Obitz TW, Eisenberg SR, Stamenovic D. Confined and unconfined stress relaxation of cartilage: appropriateness of a transversely isotropic analysis. J Biomech 1999; 32: 1125-30.

[29] Wang CCB, Deng JM, Ateshian GA, Hung CT. An automated approach for direct measurement of two-dimensional strain distributions within articular cartilage under unconfined compression. J Biomech Eng 2002; 124: 557-67.

[30] Charlebois M, McKee MD, Buschmann MD. Nonlinear tensile properties of bovine articular cartilage and their variation with age and depth. J Biomech Eng 2004; 126: 129-37.

[31] Huang CY, Stankiewicz A, Ateshian GA, Mow VC. Anisotropy, inhomogeneity, and tension-compression nonlinearity of human glenohumeral cartilage in finite deformation. J Biomech 2005; 38: 799-809.
[32] Elliott DM, Narmoneva DA, Setton LA. Direct measurement of the Poisson's ratio of human patella cartilage in tension. J Biomech Eng 2002; 124: 223-8.

[33] Wilson W, van Donkelaar CC, van Rietbergen B, Huiskes RA fibril-reinforced poroviscoelastic swelling model for articular cartilage. J Biomech 2005; 38: 1195-204.

[34] Biot MA. Theory of elasticity and consolidation for a porous anisotropic solid. J Appl Physics 1955; 26: 182-5.

[35] Spencer AJM. Deformations of Fibre-reinforced Materials. Oxford University Press: Oxford; 1972.

[36] Hill R. Aspects of invariance in solid mechanics. Adv Appl Mechanics 1978; 18: 1-75.

[37] Storakers B. On material representation and constitutive branching in finite compressible elasticity. J Mech Phys Solids 1986; 34: 12545 .

[38] Fung YC. Biomechanics: Mechanical Properties of Living Tissues. Springer-verlag: New York; 1993.

[39] Mankin HJ, Thrasher AZ. Water content and binding in normal and osteoarthritic human cartilage. J Bone Jt Surg 1975; 57A: 76-80.

[40] Venn M, Maroudas A. Chemical composition and swelling of normal and osteoarthrotic femoral head cartilage I: Chemical composition. Ann Rheum Dis 1977; 36: 121-9.

[41] Speer DP, Dahners L. Collagenous architecture of articular cartilage - correlation of scanning electron microscopy and polarized light microscopy observations. Clin Orthop Relat Res 1979; 139: $267-75$

[42] Aspden RM, Hukins DWL. Collagen organization in articular cartilage, determined by X-ray diffraction, and its relationship to tissue function. Proc R Soc Lond B 1981; 212: 299-304.

[43] Clark JM. Variation of collagen fiber alignment in a joint surface - a scanning electron-microscope study of the tibial plateau in dog, rabbit, and man. J Orthop Res 1991; 9: 246-57.

[44] Lei FL, Szeri AZ. Inverse analysis of constitutive models: biological soft tissues. J Biomech 2007; 40: 936-40. 